\begin{tabular}{|c|c|}
\hline Title & Quantum mechanical expansion of a magnetized particle in the presence of a field particle for magnetic fusion plasmas \\
\hline Author(s) & Chan, Poh Kam; Oikawa, Shun-ichi; Kosaka, W ataru \\
\hline Citation & $\begin{array}{l}\text { International journal of applied electromagnetics and mechanics, 52(3-4), 1237-1244 } \\
\text { https://doi.org/10.3233/JA E-162204 }\end{array}$ \\
\hline Issue Date & $2016-12-29$ \\
\hline Doc URL & http:/hdl.handle.net/2115/64561 \\
\hline Rights & The final publication is available at IOS Press through http://dx.doi.org/10.3233/JA E-162204 \\
\hline Type & article (author version) \\
\hline File Information & ManuscriptFinalV ersionB.pdf \\
\hline
\end{tabular}

Instructions for use 


\title{
Quantum Mechanical Expansion of a Magnetized Particle in the Presence of a Field Particle for Magnetic Fusion Plasmas
}

\author{
Poh Kam Chan, ${ }^{a, 1}$ Shun-ichi Oikawa ${ }^{\mathrm{b}}$ and Wataru Kosaka ${ }^{\mathrm{a}}$ \\ ${ }^{a}$ Graduate School of Engineering, Hokkaido University, N-13, W-8, Sapporo 060-8628, Japan \\ ${ }^{\mathrm{b}}$ Faculty of Engineering, Hokkaido University, N-13, W-8, Sapporo 060-8628, Japan
}

\begin{abstract}
The two-dimensional time-dependent Schrödinger equation, for a magnetized proton in the presence of a fixed field particle and of a uniform magnetic field is numerically solved. In the relatively high-speed case, the fast-speed proton has the similar behaviors to those of classical ones. However, in the extension of time, the relatively high-speed case shows similar behavior to the low-speed case: the cyclotron radii both in mechanical momentum and position are appreciably decreasing with time. However, the kinetic energy and the potential energy do not show appreciable changes. This is because of the increasing variances, i.e. uncertainty, both in momentum and position. The increment in variance of momentum corresponds to the decrement in the magnitude of mechanical momentum in a classical sense: Part of energy is transferred from the directional (classical kinetic) energy to the uncertainty (quantum mechanical zero-point) energy.
\end{abstract}

Keywords: uncertainty, field particle, uniform magnetic field, quantum mechanical expansion, anomalous diffusion

\section{Introduction}

Quantum mechanical application for charged particle is gaining attention by many researchers recently [1-3]. One of the interesting charged particle studies is conducted by Jüngel et al. [4-5], which showed the transient of quantum drift-diffusion equations using quasi-neutral limit for charged particle. However, later on, not much study is done on the quantum mechanical expansion study in fusion plasmas. In this paper, quantum mechanical expansion of a charged particle is investigated.

In Ref. [6], it is shown that the quantum mechanical variance in position may reach the square of the interparticle separation in a time interval of the order of $10^{-4} \mathrm{sec}$ for typical magnetically confined fusion plasmas with a number density of $n \sim 10^{20} \mathrm{~m}^{-3}$ and a temperature of $T \sim 10 \mathrm{keV}$. After this time the wavefunctions of neighboring particles would overlap, as a result the conventional classical analysis may lose its validity. Plasmas may behave like extremely-low-density liquids, not gases, since the size of each particle is of the same order of the interparticle separation.

In Refs. [7-9], the authors have also shown that for distant encounters in typical fusion plasmas of a temperature $T \sim 10 \mathrm{keV}$ and $n=10^{20} \mathrm{~m}^{-3}$, the average potential energy $\langle U\rangle \sim 30 \mathrm{meV}$ is as small as the uncertainty in energy $\Delta E \sim 40 \mathrm{meV}$, and for a magnetic field $B \sim 3 \mathrm{~T}$, the spatial size of the wavefunction in the plane perpendicular to the magnetic field is as large as the magnetic length $\sigma_{B} \sim 10^{-8} \mathrm{~m}$ which is much larger than the typical electron wavelength $\lambda_{e} \sim 10^{-11} \mathrm{~m}$, and is around one-tenth of the average interparticle separation $\Delta \ell=n^{-1 / 3}$. 
In this paper, two types of fixed field particle, positive and negative charges, are considered, which create a scalar potential on a proton. In section 2, the quantum mechanical expansion of a proton due to repulsive force is shown. In section 3, attractive force case is shown. Section 4 summarizes the study.

\subsection{Schrödinger equation}

In this paper, the two-dimensional Schrödinger equation for a wavefunction $\psi$ at position $\boldsymbol{r}$ and time $t$ is solved,

$$
\mathrm{i} \hbar \frac{\partial \psi}{\partial t}=\left[\frac{1}{2 m}(-\mathrm{i} \hbar \nabla-q A)^{2}+q V\right] \psi
$$

where $V$ and $\boldsymbol{A}$ stand for the scalar and vector potentials, $m$ and $q$ the mass and electric charge of the particle under consideration, $\mathrm{i} \equiv \sqrt{-1}$ the imaginary unit, and $\hbar=h / 2 \pi$ the reduced Planck constant. The initial condition for wavefunction at $\boldsymbol{r}=\boldsymbol{r}_{0}$ with $\boldsymbol{r}_{0}$ being the initial center of $\psi$, is given by

$$
\psi(\boldsymbol{r}, 0)=\frac{1}{\sqrt{\pi} \sigma_{B}} \exp \left[-\frac{\left(\boldsymbol{r}-\boldsymbol{r}_{0}\right)^{2}}{2 \sigma_{B}^{2}}+\mathrm{i} \boldsymbol{k}_{0} \cdot \boldsymbol{r}\right],
$$

where the magnetic length $\sigma_{B}$ is the initial standard deviation, and $\boldsymbol{k}_{0}=\left(m \boldsymbol{v}_{0}+q \boldsymbol{A}_{0}\right) / \hbar$ is the initial wavenumber vector. Here $\boldsymbol{v}_{0}$ is the initial velocity of the corresponding classical particle and $\boldsymbol{A}_{0}=\langle\boldsymbol{A}(\boldsymbol{r})\rangle$ at $t=0$. Successive over relaxation (SOR) scheme for time integration is adopted in this calculation. The spatial discretization for the two-dimensional finite difference method (FDM) needs to be sufficiently small to satisfy

$$
\Delta x \sim \Delta y \leq \frac{1}{k_{0}}=\frac{\lambda_{0}}{2 \pi},
$$

where $\lambda_{0}$ is the de Broglie wavelength. Calculation is done on a GPU [10-13] (Nvidia GTX-580: 512cores/3GB@1.54GHz), using CUDA [14,15].

In the absence of the field particle, the time dependent operator for position $\hat{x}(t)$ and $\hat{y}(t)$ in the presence of a uniform magnetic field with a Landau gauge [16] of $A_{x}=-B y, A_{y}=0, A_{z}=0$, can be easily derived as,

$$
\begin{gathered}
\hat{x}(t)=\hat{x}+\frac{\hat{P}_{y}}{q B}(1-\cos \omega t)+\left(\hat{y}+\frac{\hat{P}_{x}}{q B}\right) \sin \omega t, \\
\hat{y}(t)=-\frac{\hat{P}_{x}}{q B}+\left(\hat{y}+\frac{\hat{P}_{x}}{q B}\right) \cos \omega t+\frac{\hat{P}_{y}}{q B} \sin \omega t,
\end{gathered}
$$

where $\omega \equiv q B / m$ is the cyclotron frequency, $\hat{P}_{x}=-\mathrm{i} \hbar \partial_{x}$ is the $x$-component of the momentum operator and $\hat{P}_{y}=-\mathrm{i} \hbar \partial_{y}$ is the $y$-component of the momentum operator. However, in the presence of a field particle, the time dependent operator and its expectation value of the position of the particle is difficult to be derived. In this paper, the expectation value of the position of the particle in the presence of a field particle is numerically shown, in which the uncertainty of particle tends to expand and eventually becomes almost uniformly distributed along the classical cyclotron orbit. 


\subsection{Electrostatic potential due to a field particle}

Here the field particle is a quantum-mechanical particle, whose center is assumed to be at the origin with the wavefunction $\psi_{\mathrm{f}}$ similar to that given in Eq. (2), but is fixed in space and time, as

$$
\psi_{\mathrm{f}}(r)=\frac{\exp \left(-\frac{x^{2}+y^{2}}{2 \sigma_{B}^{2}}\right)}{\sqrt{\pi \sigma_{B}^{2}}} \times \frac{\exp \left(-\frac{z^{2}}{2 \sigma_{z}^{2}}\right)}{\sqrt{\sqrt{\pi} \sigma_{z}}},
$$

where $\sigma_{z}^{2}$ is the variance in position in $z$-direction, i.e., along the magnetic field. In magnetically confined fusion plasmas, $\sigma_{z} \sim h / m v_{0} \ll \sigma_{B}$ holds, so that the square of the second factor can be approximately the same as a Dirac delta function $\delta(z)$ centered at $z=0$. Thus the electrostatic potential $V_{\mathrm{f}}$ in the $x-y$ plane, due to the distributed charge is given by

$$
V_{\mathrm{f}}(R) \simeq \frac{q_{\mathrm{f}}}{4 \pi \varepsilon_{0} \sigma_{B}^{2}} \frac{4}{\pi} \int_{0}^{\infty} \frac{R^{\prime} e^{-\left(R^{\prime} / \sigma_{B}\right)^{2}}}{R+R^{\prime}} K(M) d R^{\prime},
$$

where $R=\sqrt{x^{2}+y^{2}}, q_{\mathrm{f}}$ is an electric charge of the field particle, $\varepsilon_{0}$ is the vacuum permittivity, and $K(M)$ is the complete elliptic integral of the first kind with the parameter $M$ being defined as $M \equiv 4 R R^{\prime} /\left(R+R^{\prime}\right)^{2}$ [16]. In the case of large distance $R \gg \sigma_{B}$ from the field particle, the electrostatic potential in Eq. (7) will become classical electrostatic potential, $V_{\mathrm{f}}(R)=q_{\mathrm{f}} /\left(4 \pi \varepsilon_{0} R\right)$ by a point charge $q_{\mathrm{f}}$.

\subsection{Errors in numerical calculations}

In this numerical calculation, the normalized initial speed in the range of $1 \leq v_{0} \leq 10$, which is much slower than the thermal speed of fusion plasmas, is used here due to the numerical reason. The restriction on numerical grid sizes $\Delta x$ and $\Delta y$ in Eq. (3) has demanded a lot of computer memory for a fast particle. The required grid sizes need to be much smaller than the de Broglie wavelength and inversely proportional to the particle speed. In fusion plasmas, these combinations of initial speed range $v_{0}$ and electric charge $q_{\mathrm{f}}=2 \times 10^{-5} \mathrm{e}$ have the similar potential-to-kinetic-energy ratio.

The accuracy of the calculation is shown in Fig. 1, in which the energy is conserved as much as 9 digits by comparing the errors to the normalized initial energy $E=50.254$ for $v_{0}=10, E=13.458$ for $v_{0}=5$, and $E=3.892$ for $v_{0}=1$.
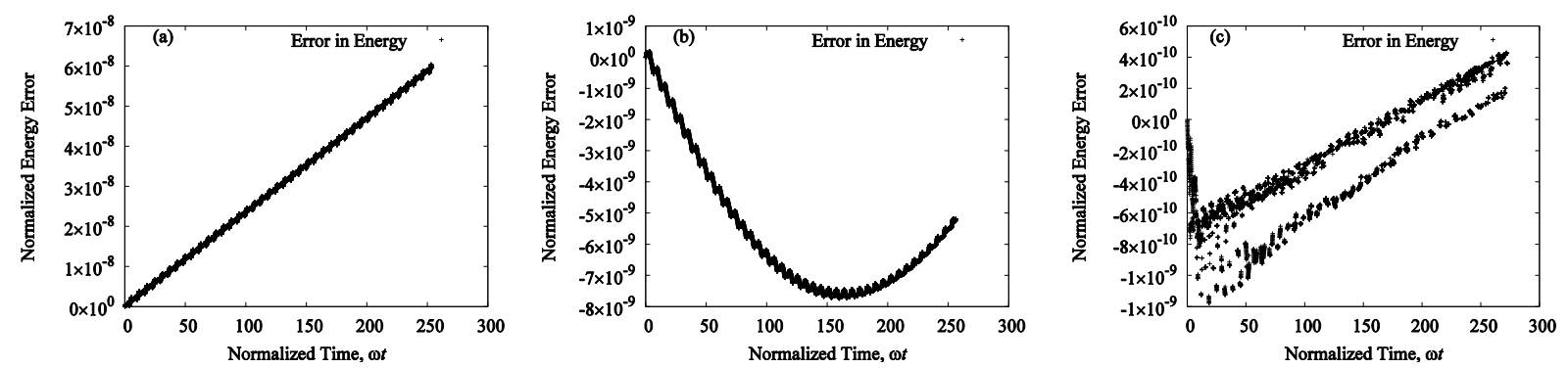

Fig. 1. Time evolution of relative errors in energy, in the presence of a fixed field particle at the origin. (a) Normalized initial speed $v_{0}=10$. (b) Normalized initial speed $v_{0}=5$. (c) Normalized initial speed $v_{0}=1$. 


\section{Case 1: Repulsive Force}

In these numerical calculations, the parameters is normalized as; mass of the particle $m=1.6722 \times 10^{-27} \mathrm{~kg}$, charge $q=1.602 \times 10^{-19} \mathrm{C}$, magnetic flux density $B=10 \mathrm{~T}$, velocity $\bar{v}=10 \mathrm{~m} / \mathrm{s}$, length $\bar{\rho}=1.04382 \times 10^{-8} \mathrm{~m}$ and time $\bar{t}=1.04382 \times 10^{-9} \mathrm{~s}$. Lengths are normalized by cyclotron radius of a proton with a speed of $10 \mathrm{~m} / \mathrm{s}$ in a magnetic field of $10 \mathrm{~T}$. The cyclotron frequency in such a case is used for normalization of the time.
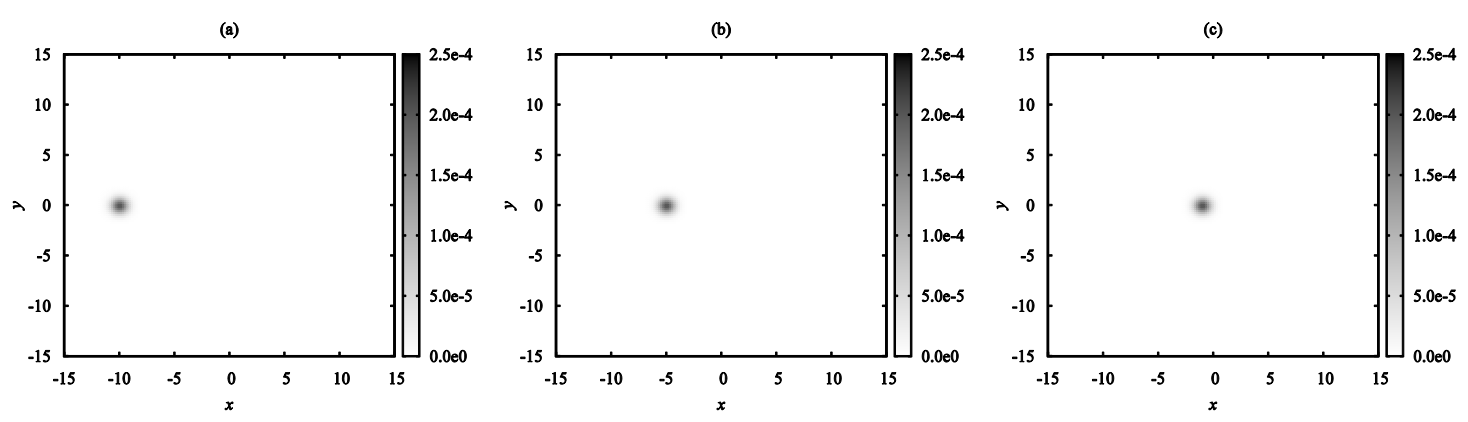

Fig. 2. Initial condition $t=0$ of probability density function (PDF) of a single charged particle, in the presence of a fixed field particle at the origin. (a) Normalized initial speed $v_{0}=10$. (b) Normalized initial speed $v_{0}=5$. (c) Normalized initial speed $v_{0}=1$.
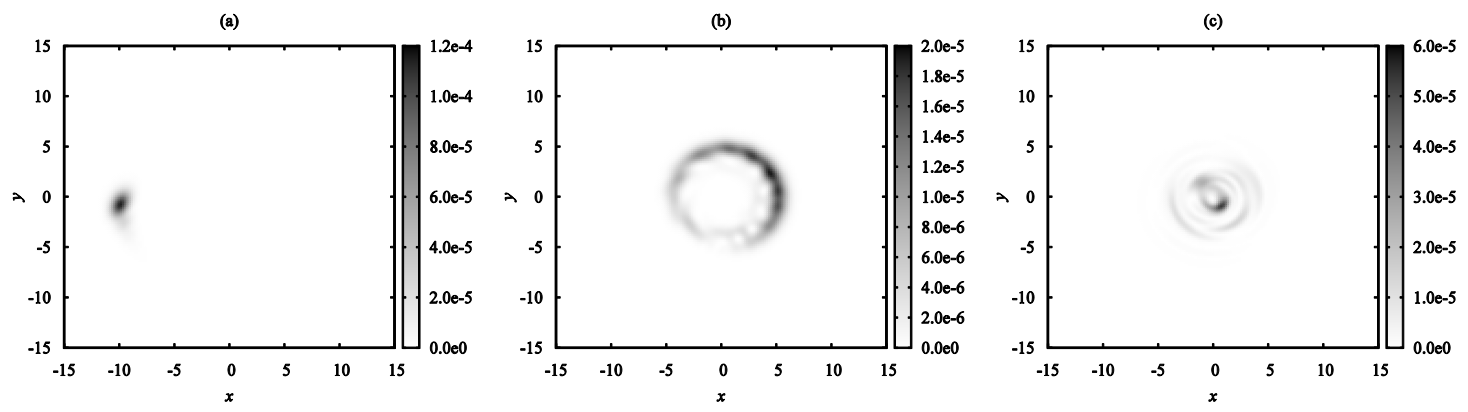

Fig. 3. The PDF of a single charged particle, in the presence of a fixed field particle at the origin, after 40 gyrations. (a) Normalized initial speed $v_{0}=10$. (b) Normalized initial speed $v_{0}=5$. (c) Normalized initial speed $v_{0}=1$.

The magnetic length for a proton in $B=10 \mathrm{~T}, \sigma_{B} \equiv \sqrt{\hbar / e B} \sim 10^{-8} \mathrm{~m}$ is a measure for the spread of a wave function in the plane perpendicular to the magnetic field. With these normalization, reduced Planck constant $\hbar \sim 0.60$, initial uncertainty in position $\sigma_{B} \equiv \sqrt{\hbar / e B} \sim \sqrt{0.60}$ and initial uncertainty in kinetic momentum $(3 / 2) \hbar e B \sim 0.91$ are order of unity. Note that the kinetic energy of a classical proton speed $v_{0} \sim 27 \mathrm{~m} / \mathrm{s}$ in $B=10 \mathrm{~T}$ corresponds to the uncertainty of the momentum. In the numerical results to be presented in the following subsections, the Schrödinger Equation is solved for the time duration of forty cyclotron rotations by a proton.

For low-speed case, $v_{0}=5$ and $v_{0}=1$, initially the probability distribution function (PDF) is a circular shape and changes to elongated shape at second gyration. This tendency grows further for each gyration. Eventually at the 40th gyration, the PDF of the particle tends to have almost uniformly distributed along the classical cyclotron orbit, as shown in Fig. 3(b), in which the width of the distribution is nearly the magnetic length of $\sigma_{B}=\sqrt{\hbar / q B}$. 


\section{$2.1 \quad$ Expectation values and variances}

The time evolution of total energy $E=K+U$, kinetic energy $K=\left\langle(m v)^{2}\right\rangle / 2 m$ and the potential energy $U=\langle q V\rangle$ is shown in Fig. 4 for initial speed of $v_{0}=10, v_{0}=5$ and $v_{0}=1$. In quantum mechanical point of view, kinetic energy is the sum of directional energy and uncertainty energy. Referring to Fig. 4(a), for high speed case, $v_{0}=10$ the particle shows the similar behavior to the classical one. Only small amount of directional energy is converted to uncertainty energy. However, for the slow particle-cases, $v_{0}=5$ and $v_{0}=1$ it is shown in Fig. 4(b,c) that the directional energy is converted to uncertainty energy after some time. Comparing with the same amount of gyrations, this phenomenon is significant for slower particle-cases.

Figure 5 shows the time evolution of normalized variance of momentum $\sigma_{P}^{2}=\left\langle(m v)^{2}\right\rangle-\langle m v\rangle^{2}$, and that of position $\sigma_{r}^{2}=\left\langle\boldsymbol{r}^{2}\right\rangle-\langle\boldsymbol{r}\rangle^{2}$, for high and low speed particle-cases. Figure 6 compare the expectation value of mechanical momentum $\langle m v\rangle$ and Fig. 7 is the comparison of expectation value of position $\langle\boldsymbol{r}\rangle$ for high and low speed particle-cases.

In the case of high speed particle, $v_{0}=10$, the trajectory in the phase space $\langle\boldsymbol{r}, \boldsymbol{p}\rangle$ is similar to the classical one and the variance in momentum and position oscillate with almost constant amplitudes [10]. However, the trajectories both in the momentum space and position in space are gradually decreasing in respective in cyclotron radii. In the meantime, the variance oscillations show significant increase in variance $\Delta \sigma^{2}$.

In the longer period of evolutions, this phenomena have the similar outcome to the lower speed case, $v_{0}=5$. It is shown that significant decrement in radii with time for both trajectories in momentum space and the configuration space. At the same time, the variances in momentum and configuration space grow with time and reach saturation. The saturation stage reached when all the directional energy are being converted to uncertainty energy.
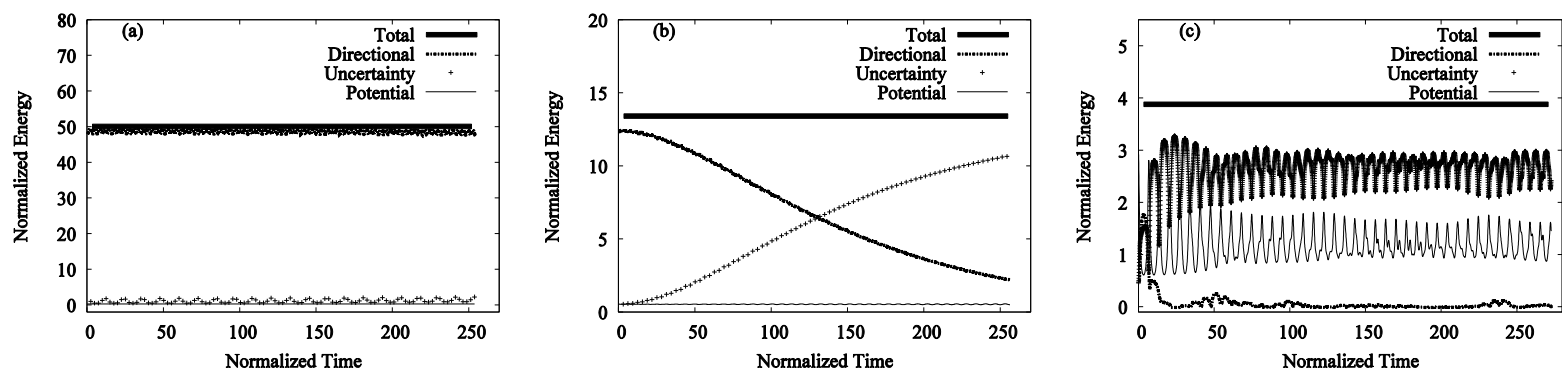

Fig. 4. Time evolution of normalized energies for 40 gyrations. (a) Normalized initial speed $v_{0}=10$ and normalized initial energy, $E=50.254$. (b) Normalized initial speed $v_{0}=5$ and normalized initial energy, $E=13.458$. (c) Normalized initial speed $v_{0}=1$ and normalized initial energy, $E=3.892$.
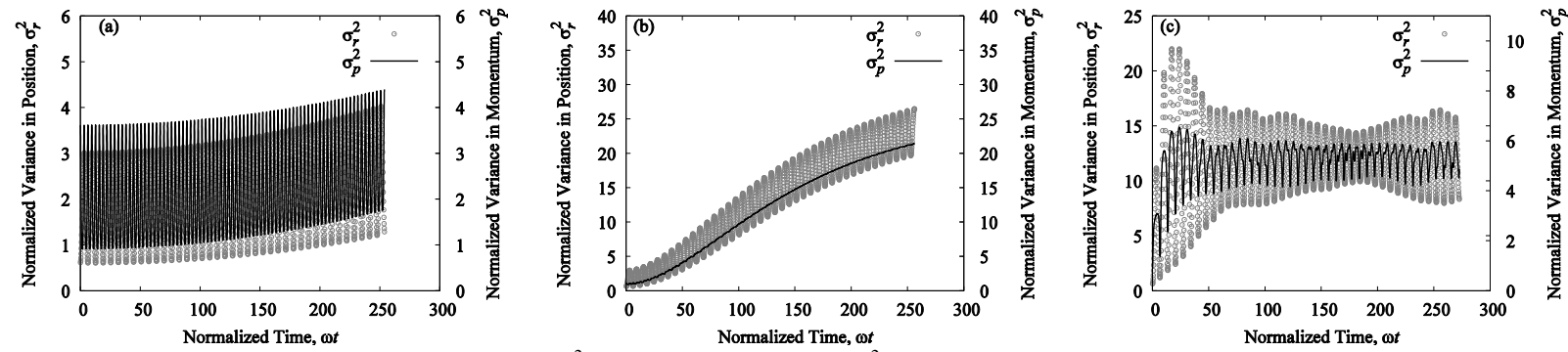

Fig. 5. Normalized variance in position $\sigma_{r}^{2}$ and total momentum $\sigma_{P}^{2}$, for 40 gyrations. (a) Normalized initial speed $v_{0}=10$.

(b) Normalized initial speed $v_{0}=5$. (c) Normalized initial speed $v_{0}=1$.

In the low speed case, $v_{0}=1$, most of the directional energy is converted to uncertainty energy in the first 10 gyrations. Corresponding to this phenomenon, the variance in momentum and position reach 
saturation at the same time. However, due to repulsion by field particle, there are large amplitude oscillation in kinetic and potential energy. The particle gyration is not in a fixed orbit as shown in Fig. 7(c).

The decrements in the magnitude of momentum $\langle m v\rangle$ as shown in Fig. 6 correspond to the increment in variance of momentum

$$
\Delta \sigma_{P}^{2}=\left\langle(m v)^{2}\right\rangle-\langle m v\rangle^{2}-(3 / 2) \hbar q B,
$$

as shown in Fig. 5. As the results of conservation of energy, part of the energy is transferred form directional energy to uncertainty or the zero-point energy.
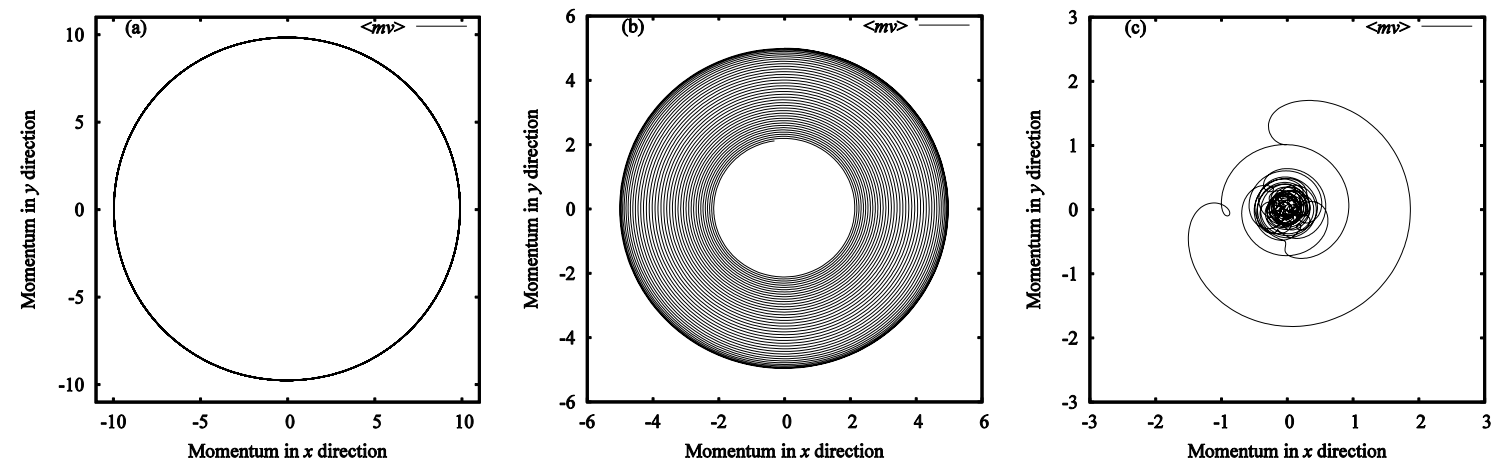

Fig. 6. Normalized expectation values of momentum $\boldsymbol{p}=\langle m \boldsymbol{v}\rangle$ after 40 gyrations. (a) Normalized initial speed $v_{0}=10$ and normalized initial momentum $\left(m u_{0}, m v_{0}\right)=(0,10)$. (b) Normalized initial speed $v_{0}=5$ and normalized initial momentum $\left(m u_{0}, m v_{0}\right)=(0,5)$. (c) Normalized initial speed $v_{0}=1$ and normalized initial momentum $\left(m u_{0}, m v_{0}\right)=(0,1)$.
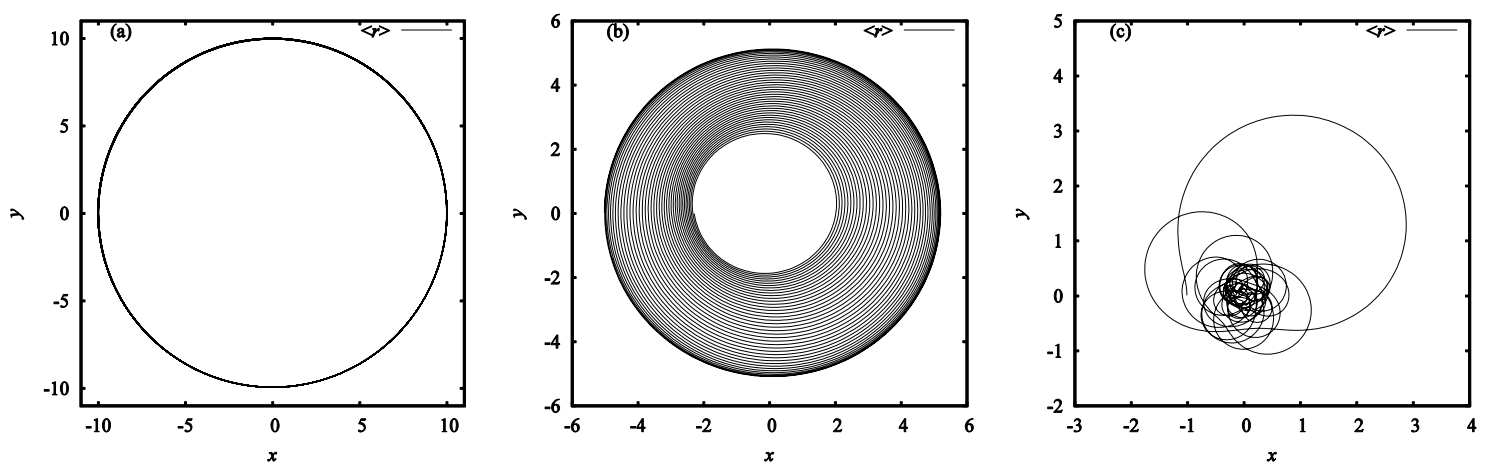

Fig. 7. Normalized expectation position $\langle\boldsymbol{r}\rangle$ after 40 gyrations. (a) Normalized initial speed $v_{0}=10$ and initial position $(-10,0)$.

(b) Normalized initial speed $v_{0}=5$ and initial position $(-5,0)$. (c) Normalized initial speed $v_{0}=1$ and initial position $(-1,0)$.

\section{Case 2: Attractive Force}

In the previous section, the study on quantum mechanical expansion for a proton which gyrates around a fixed positive charge is discussed. It is interesting to note that for repulsive force the radii of gyration decrease with gyration cycle due to directional energy conversion to uncertainty energy. In this section, further study is conducted for a proton which gyrates around a fixed negative charge which creating an attractive force. 

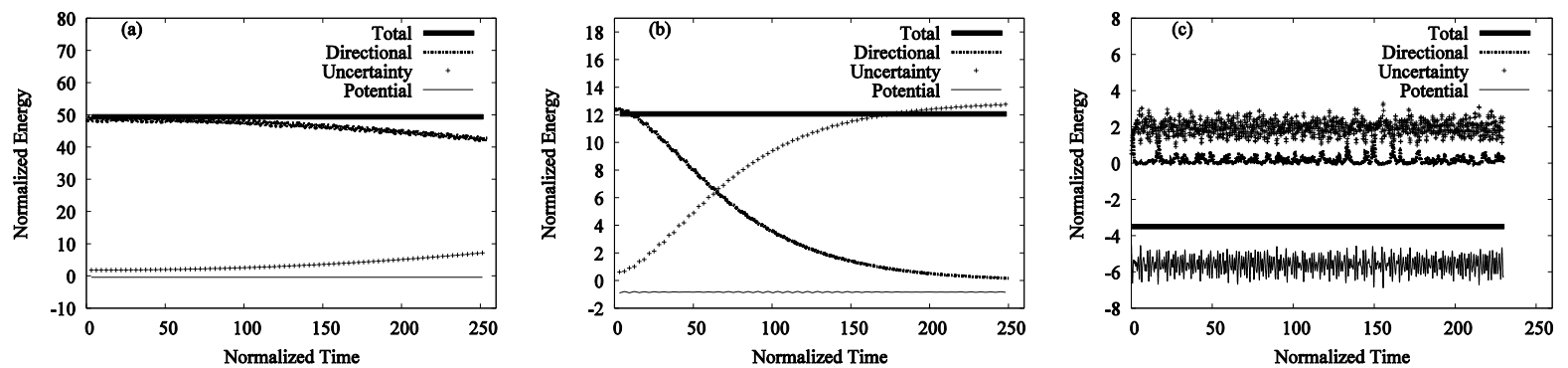

Fig. 8. Time evolution of normalized energy for 40 gyrations. (a) Normalized initial speed $v_{0}=10$ and initial normalized energy, $E=49.592$. (b) $v_{0}=5$ with $E=12.119$. (c) $v_{0}=1$ with $E=-3.457$.

In Fig. 8, the time evolution of normalized energy for 40 gyrations is shown. The energy conversion due to the negative field particle (attractive force) is shown in Fig. 8, while the positive charged field particle case (repulsive force) was shown in Fig. 4. It is shown that the quantum mechanical expansion of a particle has similar behaviors for both attractive and repulsive force cases. However, in the comparison with the same 40 gyrations cycle time between attractive force case and repulsive force case, the attractive force case shows faster conversion of directional energy to uncertainty energy.

\section{Summary}

The two-dimensional time-dependent Schrödinger equation for a magnetized proton in the presence of a fixed positive or negative field particle in the presence a uniform magnetic field is solved in this paper. In the relatively high-speed case of $v_{0}=10$, the behaviors are similar to those of classical ones. However, in the extension of time, even the fast particle behave like the low-speed case of $v_{0}=5$, where the magnitudes both in momentum $m v=|m v|$ and position $r=|\boldsymbol{r}|$ are appreciably decreasing with time. The kinetic energy $K=m\left\langle\boldsymbol{v}^{2}\right\rangle / 2$ and the potential energy $U=\langle q V\rangle$ do not show appreciable changes except for a small amplitude oscillation in high speed case, because of the increasing variances, i.e. uncertainty, both in momentum and position.

The increment in variance of momentum $\Delta \sigma_{P}^{2}$ corresponds to the decrement in the magnitude of momentum $\langle m v\rangle$ : Part of energy is transferred from the directional (classical kinetic) energy to the uncertainty (quantum mechanical zero-point) energy. Such an energy conversion is faster for attractive force case, e.g. electron-proton interaction, than the repulsive force case. For example, the quantum mechanical particle energy conversion in magnetically confined plasmas reduces the electron kinetic or mechanical speed, leading to the reduction in the relative speeds to ions including protons, deuterons and tritons. This should result in the enhanced recombination rate of an electron with a proton or its isotopes to form a hydrogen atom which is electrically neutral and is free to escape from the confining magnetic field. In summary, quantum-mechanical analyses are necessary even for fast charged particles as long as their long time behavior is concerned in the presence of a magnetic field.

\section{Acknowledgements}

The authors would like to thank Prof. S. Tomioka, Prof. Y. Matsumoto and Prof. Emeritus M. Itagaki for their fruitful discussions on the subject. Part of the SOR coding for a GPU was done by Dr. R. Ueda. This research was partially supported by a Grant-in-Aid for Scientific Research (C), Japan, 21560061.

\section{References}

[1] G. Loeper and A. Vasseur, Electric turbulence in a plasma subject to a strong magnetic field, Asymptotic Analysis, Vol. 40, no. 1 (2004), 51-65. 
1244 P.K. Chan et al. / Quantum mechanical expansion of a magnetized particle in the presence of a field particle

[2] K. Trojanowski, Properties of Quantum Particles in Multi-Swarms for Dynamic Optimization, Fundamenta Informaticae, Vol. 95, no. 2-3 (2009), 349-380.

[3] C. Negulescu, Small coherence length limit for a two-dimensional quantum transport model, Asymptotic Analysis, Vol. 49, no. 3-4 (2006), 295-329.

[4] A. Jüngel and P.J. Peng, A hierarchy of hydrodynamic models for plasmas. Quasi-neutral limits in the drift-diffusion equations, Asymptotic Analysis, Vol. 28, no. 1, pp. (2001), 49-73.

[5] A. Jüngel and I. Violet, The quasineutral limit in the quantum drift-diffusion equations, Asymptotic Analysis, Vol. 53, no. 3 (2007), 139-157.

[6] S. Oikawa, T. Shimazaki and E. Okubo, Numerical Analysis of Quantum Mechanical $\nabla B$ Drift, Plasma Fusion Res. 6, 2401058 (2011).

[7] S. Oikawa, T. Oiwa and T. Shimazaki, Preliminary Study of Uncertainty-Driven Plasma Diffusion, Plasma Fusion Res. 5, S1050 (2010).

[8] S. Oikawa, T. Oiwa and T. Shimazaki, Quantum Mechanical Plasma Scattering, Plasma Fusion Res. 5, S2024 (2010).

[9] S. Oikawa, T. Shimazaki and T. Oiwa, Preliminary Study on Uncertainty Driven Plasma Diffusion II, Plasma Fusion Res. 5, S2025 (2010).

[10] S. Oikawa, E. Okubo and P.K. Chan, Numerical Analysis of Schrodinger Equation for a Magnetized Particle in the Presence of a Field Particle, Plasma Fusion Res. 7, 2401106 (2012).

[11] P.K. Chan, S. Oikawa, and E. Okubo, Numerical Analysis of Quantum Mechanical DB Drift II, Plasma Fusion Res. 7, 2401034 (2012).

[12] S. Oikawa, P.K. Chan and E. Okubo, Plasma Fusion Res. 8, 2401142 (2013).

[13] S. Oikawa, W. Kosaka and P.K. Chan, Numerical Analysis of Quantum-Mechanical Non-Uniform E $\times \boldsymbol{B}$ Drift, Plasma Fusion Res. 9, 3401033 (2014).

[14] J. Nickolls, I. Buck, M. Garland, K. Skadron, Scalable Parallel Programming with CUDA, ACM Queue 6(2), (2008), 40-53.

[15] W. Kosaka, S. Oikawa and P.K. Chan, Numerical Analysis of Quantum-Mechanical Non-uniform $\boldsymbol{E} \times \boldsymbol{B}$ Drift: Non-uniform electric field, to be appeared in International Journal of Applied Electromagnetics and Mechanics.

[16] L.D. Landau and E.M. Lifshitz, Quantum Mechanics: Nonrelativistic Theory, 3rd ed., translated from the Russian by J.B. Sykes and J.S. Bell (Pergamon Press, Oxford, 1977).

[17] M. Abramowitz and I.A. Stegun, Handbook of Mathematical Functions (Dover, 1965). 